\title{
Temporal Domain Group Sparse Representation Based Cloud Removal for Remote Sensing Images
}

\author{
Xinghua $\mathrm{Li}^{1}$, Huanfeng Shen ${ }^{1,2(\bowtie)}$, Huifang $\mathrm{Li}^{1}$, \\ and Qiangqiang Yuan ${ }^{3}$ \\ ${ }^{1}$ School of Resource and Environmental Sciences, \\ Wuhan University, Wuhan, China \\ \{lixinghua5540, shenhf, huifangli\}@whu.edu.cn \\ ${ }^{2}$ Collaborative Innovation Center of Geospatial Technology, \\ Wuhan University, Wuhan, China \\ ${ }^{3}$ School of Geodesy and Geomatics, Wuhan University, Wuhan, China \\ yqiang86@gmail.com
}

\begin{abstract}
The reconstruction of the missing information of optical remote sensing images contaminated by unwanted cloud has attracted a great deal of attention. However, in practice, cloud removal is a challenging problem. In this paper, we propose to reconstruct the missing information by temporal domain group sparse representation. With the help of temporal normalization, the temporal complementation of multitemporal remote sensing images is strengthened. The group sparse representation, which seeks similar patches from the temporal domain, is then applied to recover the missing information. The experiments demonstrated that the proposed method is both quantitatively and qualitatively effective.
\end{abstract}

Keywords: Cloud removal · Group sparse representation $\cdot$ Image reconstruction $\cdot$ Passive remote sensing $\cdot$ Remote sensing images $\cdot$ Temporal domain

\section{Introduction}

Since remote sensing observation is multi-scale, multispectral, multitemporal, and low-cost, it has become one of the most frequently used and most powerful approaches to understanding and investigating the earth. However, with regard to passive remote sensing instruments, the information acquisition of the underlying surface is inevitably occluded when they are subject to a cloudy atmosphere. For example, on average, at any one time, approximately $35 \%$ of the global land surface is obscured by clouds [1], and in some individual countries, the cloud cover rate can be much higher. When clouds are not the research focus, they are detrimental. In order to better investigate our planet, research into cloud removal for remote sensing images is of great importance.

Cloud removal can be particularly difficult when there is a large area of cloud cover. In such a situation, the spatial domain based methods (e.g., partial differential equation based [2], maximum a posteriori based [3]), and the spectral domain based 
methods (e.g., quantitative image restoration [4], robust M-estimator multiregression [5]) are often ineffective. As a result, the temporal domain based methods have become more popular in the research community. Temporal replacement is the most simple and basic type of temporal domain based method, and it can be classified into direct and indirect replacement. In terms of direct replacement, the cloud-contaminated pixels are replaced by their counterparts from another time, but the time interval must be sufficiently short that the temporal difference can be ignored. The representative methods include image mosaicing [6], the maximum value composite (MAC) technique [7], and optimal pixel selection [1]. In contrast, when the temporal difference is great, indirect replacement is required. Unlike direct replacement, a transformation is needed to reduce the temporal difference. Temporal fitting [8], for example, belongs to this kind of approach. In brief, temporal replacement is simple and widely used; however, it ignores the local changes with time.

Temporal replacement is usually suitable for a short time series of remote sensing images; in contrast, when the time series is long, more effective methods are required. The most noteworthy are the temporal filter methods, in which the two most common and classical methods (sliding window filter) are the best index slope extraction (BISE) method [9] and the adaptive Savitzky-Golay (SG) filter [10]. In addition, the function-based curve fitting methods are also effective, e.g., the asymmetric Gaussian (AG) approach [11]. The third type of filter method is the frequency filter, such as the famous harmonic analysis of time series (HANTS) method [12]. To some degree, a long time series is a double-edged sword, from which the temporal filter methods both benefit and are impaired.

In addition to the above methods, in consideration of the advantage over signal representation, sparse representation based methods have been the subject of much attention in the field of missing information reconstruction. The basic idea of sparse representation is to represent the signal by a linear combination of a few elements from a dictionary, in which the elements are called atoms. Lorenzi et al. [13] proposed to reconstruct the cloud-contaminated region by two geo-registered images from different periods, under a compressive sensing perspective, and the results were promising. $\mathrm{Li}$ et al. [14] then proposed multitemporal dictionary learning to further utilize the temporal complementation in a longer time series. Recently, Li et al. [15] made a comparison between spectral complementation and temporal complementation in the framework of sparse representation, and the authors concluded that the results are generally better when spectral complementation is available. In general, the aforementioned sparse representation methods can obtain good results; however, as Zhang et al. [16] noted, these methods just make use of the local sparsity while ignoring the nonlocal similarity [17]. In order to make better use of the temporal sparsity and nonlocal similarity, we propose temporal group sparse representation to reconstruct the missing information of optical remote sensing images.

The rest of this paper is organized as follows. Section 2 introduces the proposed group sparse representation algorithm, which is followed by the validated experiments in Sect. 3. Section 4 concludes the paper. 


\section{Algorithm}

In this section, the proposed algorithm is introduced. It includes three main parts: temporal normalization and permutation, temporal similar patch search, and temporal group sparse representation.

\subsection{Temporal Normalization and Permutation}

Suppose that $T$ multitemporal remote sensing images $\left\{I_{t}\right\}_{t=1}^{T} \in \mathbb{R}^{m \times n}$ are geo-registered. For brevity, we first assume that each image $I_{t}$ is two-dimensional. Specifically, $I_{1}$ is contaminated by clouds, and the corresponding area is denoted by $\Omega$, and the rest of the area (cloud-free) is denoted by $\bar{\Omega}$. For the other $\left\{I_{t}\right\}_{t=2}^{T}$, the area of each image is also divided into $\Omega$ and $\bar{\Omega}$, as $I_{1}$ is. Hereafter, $I_{1}$ is called the target image (cloudcontaminated) and $\left\{I_{t}\right\}_{t=2}^{T}$ are the reference images (cloud-free). As the shooting times are different, differences exist between the target image and the reference images. To reduce the differences, temporal normalization is adopted. We assume that the reference images are linearly correlated to the target image. The linear relationships can then be obtained by their shared good region $\bar{\Omega}$. Finally, based on the derived linear relationships, the reference images are temporally normalized. Mathematically, the linear relationships are modeled by:

$$
I_{1}(\bar{\Omega})=a_{t} I_{t}(\bar{\Omega})+b_{t}, \quad t=2,3, \cdots, T
$$

where $I_{t}(\bar{\Omega})$ denotes the pixels in region $\bar{\Omega}$ of $I_{t}$, and $a_{t}$ and $b_{t}$ are the normalized parameters. With a least-squares solver, each pair of $a_{t}$ and $b_{t}$ are easily obtained. Subsequently, $\left\{I_{t}\right\}_{t=2}^{T}$ are normalized by:

$$
I_{t}^{n}(\bar{\Omega} \cup \Omega)=a_{t} I_{t}(\bar{\Omega} \cup \Omega)+b_{t}, \quad t=2,3, \ldots, T
$$

where $I_{t}^{n}$ represents the normalized reference images.

To better utilize the temporal correlation between the target and reference images, we resort to temporal permutation. As shown in Fig. 1, after temporal normalization, the same rows from different images will be grouped together chronologically. This approach is also beneficial to the temporal similar patch search, which is introduced in Sect. 2.2. If the images themselves are multispectral, the normalization and permutation is undertaken according to their corresponding spectral bands, as in the previous process.

\subsection{Temporal Similar Patch Search}

The most notable characteristic of temporal group sparse representation is the grouping of the similar patches. The similar patches represent the so-called nonlocal similarity. In this section, we describe how to search for the similar patches. After the temporal 


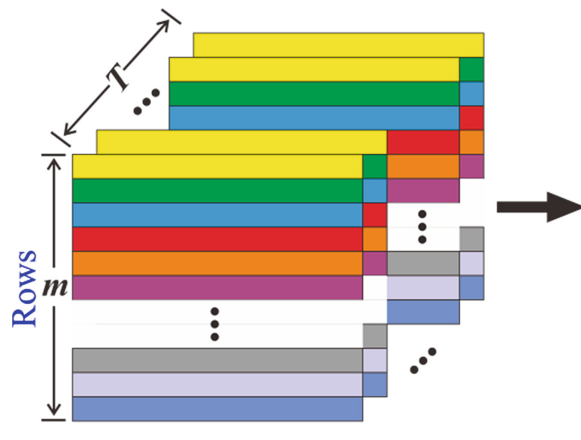

Target and reference images

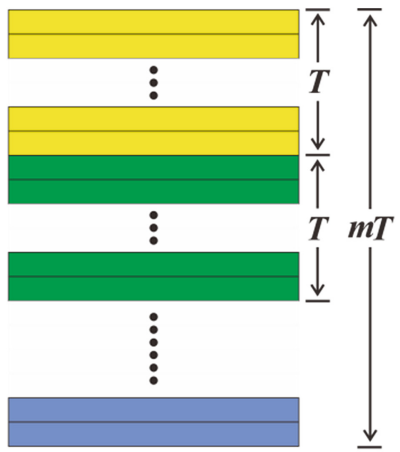

Permutation result

Fig. 1. Temporal permutation.

permutation, the result is denoted by a column-stacked $x \in \mathbb{R}^{m n T}$. Let $x_{k} \in \mathbb{R}^{B}$ denote the extracted patch from $x$ with the size of $\sqrt{B} \times \sqrt{B}$ at position $k$, and $k=1,2, \ldots, N$, where $N$ is the total patch number. We let

$$
x_{k}=P_{k}(x)
$$

where $P_{k}(\bullet)$ represents the operator which extracts the $k$-th patch, and we let it be a vector. Correspondingly, $P_{k}^{-}(\bullet)$ means to put back the vector into its original patch position in the image domain.

The similarity criteria between vectors include the Euclidean distance, the cosine distance, the correlation coefficients (CCs), and so on. Based on our experiments, the $\mathrm{CC}$ is better than most other similarity criteria. As a result, the similar patches are determined according to the $\mathrm{CC}$. The higher the $\mathrm{CC}$, the more similar to the reference patch the current patch is. Taking $x_{i}$ and $x_{j}$ as an example, their CCs are calculated by:

$$
C C_{i, j}=\frac{\sum_{B}\left(x_{i}-\mu_{x_{i}}\right)\left(x_{j}-\mu_{x_{j}}\right)}{\sqrt{\sum_{B}\left(x_{i}-\mu_{x_{i}}\right)^{2}} \sqrt{\sum_{B}\left(x_{j}-\mu_{x_{j}}\right)^{2}}}
$$

where $C C_{i, j}$ denotes the CCs of $x_{i}$ and $x_{j}$, and $\sum_{B}(\bullet)$ represents the sum of the $B$ elements. Here, we reiterate that $x$ is also contaminated by clouds. If we directly select similar patches, the wrong patches may be merged. In this situation, simple interpolation of the corrupted pixels makes a difference. Since the cloud-contaminated region is usually large, spatial domain interpolation using the corrupted image itself will obtain a poor result. Therefore, we resort to temporal interpolation. No matter how large the cloud region is, the nearest-neighbor interpolation is effective. This is also the reason that we undertake the temporal permutation described in Sect. 2.1.

In order to reduce the computational complexity, we do not search for the similar patches to $x_{k}$ in the whole of $x$. Instead, the similar patches to $x_{k}$ are searched for in the 
neighborhood of position $k$. When the $\mathrm{CC}$ is higher than the threshold $C C_{\text {threshold }}$, the patches are considered as similar patches. After all the similar patches to $x_{k}$ are found, $x_{G_{k}}$ is obtained by concatenating every similar vector column by column.

\subsection{Temporal Group Sparse Representation}

According to Sect. 2.2, we know that each patch $x_{k}$ corresponds to a group $x_{G_{k}}$. The group sparse representation means that each group $x_{G_{k}}$ can be sparsely represented by the linear combination of the atoms of dictionary $D_{G_{k}}[16]$. We let

$$
x_{G_{k}}=P_{G_{k}}(x)
$$

Similarly, $P_{G_{k}}^{-}(\bullet)$ is the inverse operation of $P_{G_{k}}(\bullet)$. As in [16], the group sparse representation is expressed as:

$$
x=D_{G} \circ \alpha_{G}=\sum_{k=1}^{N} P_{G_{k}}^{-}\left(D_{G_{k}} \alpha_{G_{k}}\right) \cdot / \sum_{k=1}^{N} P_{G_{k}}^{-}\left(E_{D_{k} \alpha_{k}}\right)
$$

where $E_{D_{k} \alpha_{k}}$ means the same size as $D_{G_{k}} \alpha_{G_{k}}$ with all ones, and $(\bullet) . /(\bullet)$ represents the element-wise division. In fact, this equation means the average of all the overlapped patches according to their overlapped times. Given the group dictionary $D_{G_{k}}$, reconstructing the missing information is to solve:

$$
\underset{\alpha_{G}}{\arg \min }\left\|M\left(x-D_{G} \circ \alpha_{G}\right)\right\|_{2}^{2}+\lambda\left\|\alpha_{G}\right\|_{0}
$$

where $M$ is a diagonal matrix with diagonal elements consisting of 0 and 1 , with 0 representing the missing position, $\lambda$ is the regularized parameter, and $\|\bullet\|_{0}$ and $\|\bullet\|_{2}$ denote the $\ell_{0}$ and $\ell_{2}$ norms, respectively. Since (7) is NP-hard, it is substituted by the convex version:

$$
\underset{\alpha_{G}}{\arg \min }\left\|M\left(x-D_{G} \circ \alpha_{G}\right)\right\|_{2}^{2}+\lambda\left\|\alpha_{G}\right\|_{1}
$$

where $\|\bullet\|_{1}$ denotes the $\ell_{1}$ norm. According to [16], Eq. (8) is equivalent to:

$$
\underset{\alpha_{G_{k}}}{\arg \min }\left\|\alpha_{G_{k}}-\gamma_{r_{G_{k}}}\right\|_{2}^{2}+\tau\left\|\alpha_{G_{k}}\right\|_{0}
$$

where $\gamma_{r_{G_{k}}}$ is the singular value of the residual matrix $r_{G_{k}}$, and $\tau$ is the new regularized parameter. This can be solved based on either the hard or soft threshold. 


\section{Experiments}

To validate the effectiveness of the proposed temporal domain group sparse representation (TDGSR), we undertook both simulated and real data cloud removal experiments based on Moderate Resolution Imaging Spectroradiometer (MODIS) images. In the two experiments, the data were both the $250-\mathrm{m}$ resolution reflectance product of MODIS L1B, and the images were cropped to the size of $400 \times 400$ from the geo-registered images. For brevity, only one reference image was used in each experiment. For the simulated experiment, the image to be reconstructed was acquired by Terra MODIS on November 2, 2013 (some pixels of which were artificially removed), and the reference image was acquired by Aqua MODIS on November 4, 2013 , in the same geographical area. For the real data experiment, the cloud-contaminated image was acquired by Terra MODIS on December 27, 2008, and the reference image was acquired by Terra MODIS on December 18, 2008. Additionally, the parameters were set as follows: patch size $\sqrt{B}=4, C C_{\text {threshold }}=0.85$, and $\tau$ is an adaptive parameter to the number of similar patches.

\subsection{Simulated Experiment}

In the simulated experiment, the artificially corrupted image was reconstructed by direct replacement of the reference image, MT-KSVD [14], and the proposed TDGSR, respectively. Figure 2 shows the reconstruction results of the different algorithms. From the visual effect, the direct replacement [Fig. 2(c)] obtained the worst result, which

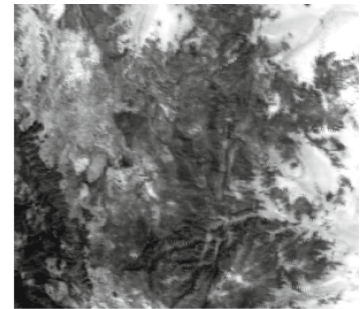

(a)

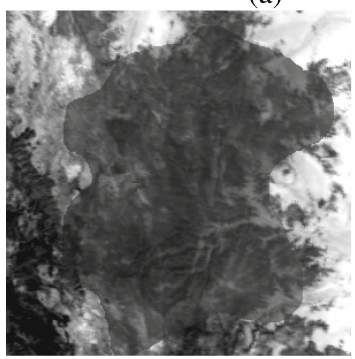

(c)

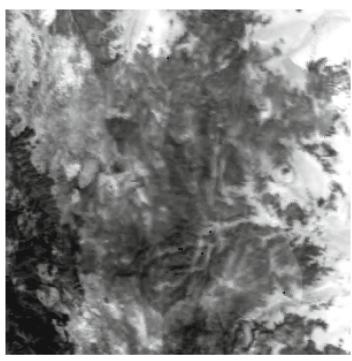

(d)

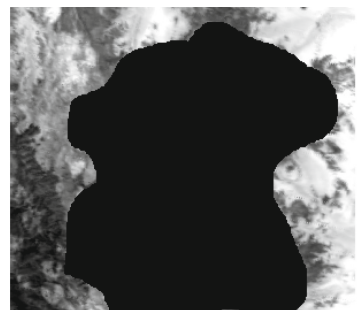

(b)

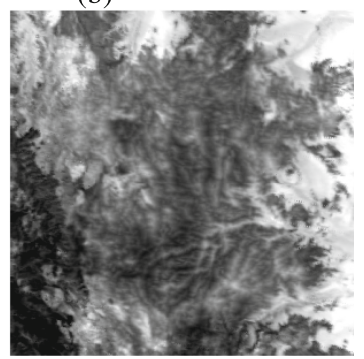

(e)

Fig. 2. Reconstruction results of the different methods. (a) Original image. (b) Corrupted image. (c) Reconstruction result using direct replacement of the reference image. (d) Reconstruction result using MT-KSVD. (e) Reconstruction result using the proposed TDGSR. 


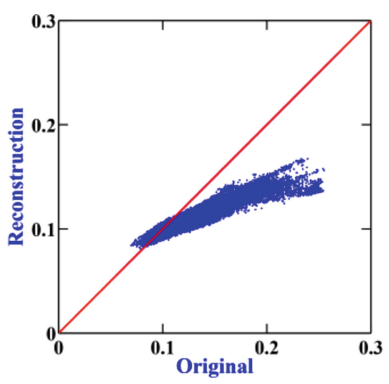

(a)

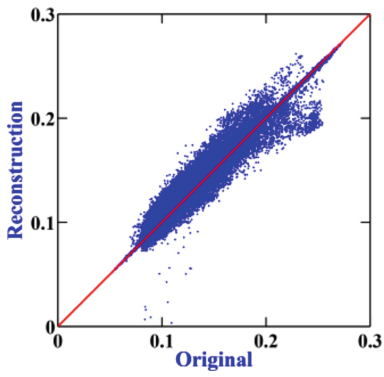

(b)

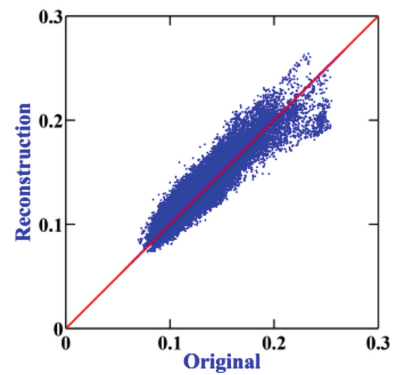

(c)

Fig. 3. Scatter plots between the original and the reconstructed images using the different algorithms. (a) Direct replacement. (b) MT-KSVD. (c) TDGSR.

shows obvious differences between the good region and the reconstructed region. Both MT-KSVD and TDGSR obtained satisfactory results, as shown in Fig. 2(d) and (e), respectively.

Since the difference is not obvious between the reconstruction results of MT-KSVD and TDGSR, scatter plots between the original and the reconstructed images are shown in Fig. 3. As before, direct replacement [Fig. 3(a)] was less effective than MT-KSVD [Fig. 3(b)] and TDGSR [Fig. 3(c)]. Moreover, the scatter plots of MT-KSVD are distributed a bit more discretely than those of TDGSR, which means that MT-KSVD performed worse than TDGSR.

In addition, we show the quantitative assessment of the reconstruction results in Table 1 by the metrics of mean absolute error (MAE), mean square error (MSE), mean relative error (MRE), and CC. Because of the length limitation, we refer the readers to our previous paper [14] for their calculation expressions. Table 1 demonstrates that MT-KSVD and TDGSR effectively reconstructed the missing information, and TDGSR performed better than MT-KSVD.

Table 1. Quantitative assessment of the reconstruction results.

\begin{tabular}{l|c|c|c|l}
\hline Method & MAE / $10^{-3}$ & MSE / $10^{-5}$ & MRE / \% & CC \\
\hline Corrupted & 66.947 & 895.612 & 52.213 & 0.6579 \\
\hline Replacement & 18.594 & 116.812 & 13.973 & 0.9058 \\
\hline MT-KSVD & 4.690 & 6.512 & 3.676 & 0.9851 \\
\hline TDGSR & 4.381 & 6.112 & 3.468 & 0.9858 \\
\hline
\end{tabular}




\subsection{Real Data Experiment}

In order to verify the cloud removal effect for real remote sensing images, we also conducted a real data experiment. As in the simulated experiment, obvious differences exist between the good part and the reconstructed part of the cloudy area in the result of direct replacement. Both MT-KSVD and TDGSR effectively reconstructed the missing information obscured by clouds, and again performed better than direct replacement (Fig. 4).

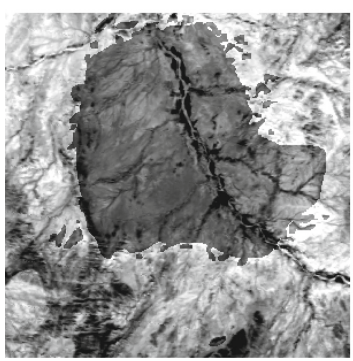

(b)

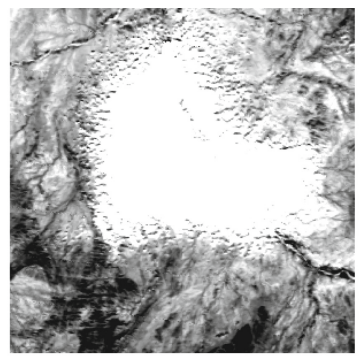

(a)

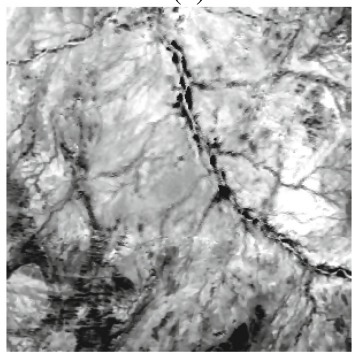

(c)

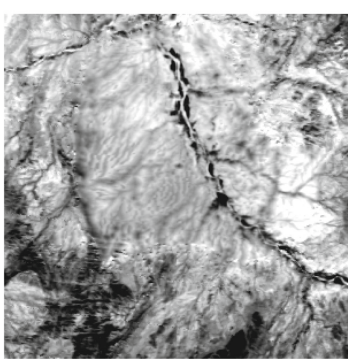

(d)

Fig. 4. Cloud removal results.

\section{Conclusions}

This paper has proposed a cloud removal algorithm for remote sensing images, using group sparse representation in the temporal domain. The main idea is to make use of the temporal sparsity and nonlocal similarity in the multitemporal images. Temporal correlation is utilized in the reconstruction process of the missing information. In order to make better sense of the temporal correlation, temporal normalization and permutation are also adopted. Simulated and real data experiments both confirmed that the proposed method is an effective approach.

Acknowledgements. This work was supported by the National Natural Science Foundation of China (NSFC) under Grant Nos. 41422108 and 41401396, the Postdoctoral Science Foundation of China under Grant No. 2014M552083, and the Fundamental Research Funds for the Central Universities of China under Grant No. 2014205020201. The authors would also like to thank the anonymous reviewers. 


\section{References}

1. Lin, C.-H., Lai, K.-H., Chen, Z.-B., Chen, J.-Y.: Patch-based information reconstruction of cloud-contaminated multitemporal images. IEEE Trans. Geosci. Remote 52(1), 163-174 (2014)

2. Barcelos, C., Batista, M.A.: Image inpainting and denoising by nonlinear partial differential equations. In: 16th Brazilian Symposium on Computer Graphics and Image Processing (SIBGRAPI), pp. 287-293. Sao Carlos, Brazil (2003)

3. Shen, H., Zhang, L.: A MAP-based algorithm for destriping and inpainting of remotely sensed images. IEEE Trans. Geosci. Remote 47(5), 1492-1502 (2009)

4. Gladkova, I., Grossberg, M.D., Shahriar, F., Bonev, G., Romanov, P.: Quantitative restoration for MODIS band 6 on aqua. IEEE Trans. Geosci. Remote 50(6), 2409-2416 (2012)

5. Li, X., Shen, H., Zhang, L., Zhang, H., Yuan, Q.: Dead pixel completion of aqua MODIS band 6 using a robust m-estimator multiregression. IEEE Geosci. Remote S. 11(4), 768-772 (2014)

6. Helmer, E., Ruefenacht, B.: Cloud-free satellite image mosaics with regression trees and histogram matching. Photogramm. Eng. Remote Sens. 71(9), 1079-1089 (2005)

7. Holben, B.N.: Characteristics of maximum-value composite images from temporal AVHRR data. Int. J. Remote Sens. 7(11), 1417-1434 (1986)

8. Zhang, J., Clayton, M.K., Townsend, P.A.: Functional concurrent linear regression model for spatial images. J. Agric. Biol. Environ. S. 16(1), 105-130 (2011)

9. Viovy, N., Arino, O., Belward, A.S.: The best index slope extraction (BISE): a method for reducing noise in NDVI time-series. Int. J. Remote Sens. 13(8), 1585-1590 (1992)

10. Savitzky, A., Golay, M.J.E.: Smoothing and differentiation of data by simplified least squares procedures. Anal. Chem. 36(8), 1627-1639 (1964)

11. Jönsson, P., Eklundh, L.: Seasonality extraction by function fitting to time-series of satellite sensor data. IEEE Trans. Geosci. Remote 40(8), 1824-1832 (2002)

12. Verhoef, W., Menenti, M., Azzali, S.: Cover a colour composite of NOAA-AVHRR-NDVI based on time series analysis (1981-1992). Int. J. Remote Sens. 17(2), 231-235 (1996)

13. Lorenzi, L., Melgani, F., Mercier, G.: Missing-area reconstruction in multispectral images under a compressive sensing perspective. IEEE Trans. Geosci. Remote 51(7), 3998-4008 (2013)

14. Li, X., Shen, H., Zhang, L., Zhang, H., Yuan, Q., Yang, G.: Recovering quantitative remote sensing products contaminated by thick clouds and shadows using multitemporal dictionary learning. IEEE Trans. Geosci. Remote 52(11), 7086-7098 (2014)

15. Li, X., Shen, H., Zhang, L., Li, H.: Sparse-based reconstruction of missing information in remote sensing images from spectral/temporal complementary information. ISPRS J. Photogramm. doi: 10.1016/j.isprsjprs.2015.03.009

16. Zhang, J., Zhao, D., Gao, W.: Group-based sparse representation for image restoration. IEEE Trans. Image Process. 23(8), 3336-3351 (2014)

17. Buades, A., Coll, B., Morel, J.M.: A non-local algorithm for image denoising. In: IEEE Computer Society Conference on Computer Vision and Pattern Recognition (CVPR), pp. 60-65, San Diego, USA (2005) 